\title{
Bilateral Basal Ganglia Lesions in Patients with Heroin Overdose: A Report of Two Cases
}

\author{
Ali Hassan ${ }^{a} \quad$ Mahdi Al Jawad $^{\mathrm{b}}$ Amna Alsaihati $^{\mathrm{a}}$ Haitham Alaithan $^{\mathrm{c}}$ \\ Faisal Al Hawaj \\ aDepartment of Emergency Medicine, King Fahd Hospital of the University, \\ Imam Abdulrahman Bin Faisal University, Al-Khobar, Saudi Arabia; ${ }^{b}$ Department \\ of Radiology, King Fahd Hospital of the University, Imam Abdulrahman Bin Faisal \\ University, Al-Khobar, Saudi Arabia; 'Department of Internal Medicine, Imam \\ Abdulrahman Bin Faisal University, Dammam, Saudi Arabia
}

\section{Keywords}

Basal ganglia $\cdot$ Coma $\cdot$ Computed tomography $\cdot$ Opioids $\cdot$ Overdose

\begin{abstract}
The prevalence of opioid use has increased worldwide. Two-thirds of deaths caused by drug overdose are opioid-related. Individuals with opioid use may present with a variety of complications. The available history in unconscious patients is often insufficient, which may cause diagnostic difficulty and delayed management. We present two cases of 54-year-old and 25year-old male patients who were brought to our emergency department after being found unconscious at home, without any known preceding event. They were in a deep coma with a Glasgow coma score of 3/15. However, their brainstem reflexes were normal. Pinpoint pupils were observed bilaterally. Brain computed tomography (CT) demonstrated the presence of bilateral basal ganglia hypodensities in both patients, in addition to multiple hypodensities scattered in the cerebral hemispheres of one patient. Toxicology screening from both patients was positive for opioids. The patients were transferred to the intensive care unit for supportive management. One patient recovered completely, whereas the other remained in a vegetative state. The presence of bilateral basal ganglia lesions in brain CT in unconscious patients should alert the physician of opioid use disorder among these patients.
\end{abstract}




\section{Introduction}

The incidence of opioid use has been increasing globally [1]. In the United States, it is estimated that approximately two million individuals have opioid addiction [2]. The morbidity and mortality due to opioid use have become an epidemic problem as almost two-thirds of deaths due to drug overdose are caused by opioids [3].

In emergency settings, individuals using opioids may present with a variety of complications, including neurologic, musculoskeletal, genitourinary, and gastrointestinal conditions [4]. Herein, we describe two patients who were in coma due to opioid overdose and in whom computed tomography (CT) demonstrated bilateral lesions involving the basal ganglia. The presence of bilateral basal ganglia lesions should be considered as a diagnostic clue for opioid overdose in unconscious patients.

\section{Case Presentation}

Case 1

A 54-year-old male patient was brought to the emergency department of our hospital by paramedics after he had been found unresponsive at his home by his family. His family had not noticed any jerky movements or classic seizure manifestations. The patient had no history of preceding trauma or headache. He had a past medical history of hypertension, diabetes mellitus, and epilepsy. The family reported that the patient was a heavy cigarette smoker, but gave no history of recreational drug use.

On presentation to the emergency department, the patient was found to have bilateral nonreactive pinpoint pupils and central cyanosis. He was afebrile, and his blood pressure, pulse rate, respiratory rate, and oxygen saturation were 90/63 mm Hg, $110 \mathrm{bpm}, 7$ breaths/ min, and $77 \%$ on room air, respectively. He had no voluntary or involuntary movements. His Glasgow coma score was 3/15 and he had intact brainstem reflexes.

The patient was resuscitated with intravenous fluids, intubated, and mechanically ventilated. Brain CT was performed and demonstrated bilateral hypodensities in the globi pallidi and genu of the internal capsule (Fig. 1). A urine toxicology screen was positive for opioids. Other drugs, including amphetamines, barbiturates, benzodiazepine, and tetrahydrocannabinol, were not detected in the screen. The results of the laboratory tests, including those for complete blood count, urea, electrolytes, and liver functions were all within normal limits.

Given the clinical and laboratory findings, the patient was administered intravenous naloxone, but this yielded no response. Subsequently, repeated doses were given until he had received a total dose of $10.4 \mathrm{mg}$, which also failed to elicit any response. No further doses were given as no naloxone vials were available.

The patient was transferred to the intensive care unit, with continued supportive care. On the following day, he regained consciousness and showed clinical improvement in terms of hemodynamic and respiratory status. He confirmed having used heroin injection. He wanted to leave against medical advice and was deemed to be competent to do so by a psychiatrist. Based on electronic medical records, the patient has not returned to the hospital to date.

Case 2

This case was a 25-year-old man with a history of heroin addiction for which he was receiving maintenance treatment with methadone. He was brought to the hospital by the 
emergency medical services after his father had found him unresponsive with a needle and syringes around him. The rest of the patient's medical history was noncontributory.

On examination, he had a Glasgow coma score of 3/15 with a blood pressure of 104/ $68 \mathrm{~mm} \mathrm{Hg}$, a heart rate of $115 \mathrm{bpm}$, a respiratory rate of 8 breaths/min, a temperature of $36.9^{\circ} \mathrm{C}$, and an oxygen saturation of $79 \%$ on room air. He had nonreactive pinpoint pupils as well as hypotonia and hyporeflexia bilaterally in both the upper and lower limbs. The rest of the examination was unremarkable.

The patient was intubated and transferred to the medical intensive care unit. He received intravenous fluid and a total dose of $4 \mathrm{mg}$ of naloxone. His condition improved as his Glasgow coma score increased to 6/15 (E2V1M3). The urine toxicology screen was positive for opioids and amphetamine. The rest of the laboratory investigations was within normal limits.

On the second day, the patient had multiples spikes of fever reaching up to $39^{\circ} \mathrm{C}$. A chest $\mathrm{X}$-ray showed infiltration in the right lower lobe. He was started on colistin and tigecycline as transtracheal culture showed drug-resistant Acinetobacter baumannii. He developed jerky movements in the lower limbs. A CT scan of the brain revealed multiple areas of subcortical and periventricular hypodensities scattered within the bilateral cerebral and cerebellar hemisphere, including the globi pallidi (Fig. 2). The electroencephalogram findings were suggestive of diffuse cerebral dysfunction consistent with cerebral encephalopathy with hypoxic etiology without epileptiform discharges. The neurology team's assessment attributed the movements to spinal myoclonus secondary to brain hypoxia. No improvement in consciousness level was noted for the next 2 weeks. The patient underwent tracheostomy and percutaneous endoscopic gastrostomy tube insertion in preparation for prolonged rehabilitation. He was discharged to a rehabilitation center in a vegetative state.

\section{Discussion}

The basal ganglia are a group of nuclei located deep within the cerebral hemispheres. These nuclei have high energy requirements and are rich in blood flow and neurotransmitters [5]. They are susceptible to selective injuries due to systemic conditions that alter cerebral metabolism.

The potential insults to the basal ganglia include toxic, metabolic, vascular, infectious, or degenerative etiologies. Examples of these conditions include cyanide or methanol poisoning, hypo- and hyperglycemia, hypertensive crises, and some inborn errors of metabolism (e.g., Leigh's syndrome and citrullinemia) [5]. Such lesions on imaging studies should be combined with careful clinical assessment to narrow the differential diagnoses and guide further investigations [5].

Bilateral basal ganglia injury has been strongly linked to carbon monoxide poisoning and was thought to be a characteristic feature of carbon monoxide poisoning. However, Yarid and Harruff [6] reported 27 cases of basal ganglia necrosis based on autopsy examinations in 2015 , none of which were related to carbon monoxide poisoning. Of these 27 cases, 10 were related to drug overdose.

In an autopsy study by Andersen and Skullerud [7] involving 100 individuals who were intravenous heroin users, it was concluded that around $5-10 \%$ of users may have had pallidal infarcts with or without other cerebral injuries. Of note, the basal ganglia changes were reported in individuals who used inhaled heroin and other opioids such as methadone [8, 9].

A study conducted by Taheri et al. [10] in 2011 on a cohort of 92 unconscious patients with opioid overdose showed that $15.2 \%$ of the patients had abnormal findings on CT scan. 
These findings included infarction, hemorrhage, cerebral edema, and basal ganglia changes in one patient. A similar study by Jamshidi et al. [11] on patients with acute opioid overdose found that abnormal brain CT findings were present in approximately $20 \%$ of patients who underwent CT for examination due to severe symptoms. Furthermore, presence of abnormal CT findings was associated with a poor prognosis.

The exact pathophysiology of basal ganglia injury in opioid users is not well understood. However, various hypotheses have been proposed, including hypoxic cerebral injury and direct neuronal toxicity [12]. Stimulation of $\mu$-opioid receptors can cause respiratory depression, hypoxia, and subsequently death [13]. Furthermore, vasospasm induced by action on $\mu$-opioid receptors may result in cerebral ischemia or infarction [13]. However, the basal ganglia were not involved in several cases of respiratory depression and cerebral hypoperfusion in opioid users [14]. Alternatively, some researchers have suggested that basal ganglia involvement is a result of neuronal toxicity specific to the basal ganglia. Pearson et al. [15] demonstrated a decrease in the number of neurons in the basal ganglia of opioid users.

Brain CT is often performed to evaluate unconscious patients, to determine the etiology of altered mental status, and to estimate the severity of brain injury. It is the investigation of choice for excluding common pathologies such as hemorrhage, infarction, or space-occupying lesions. Although the family of case 1 failed to report a history of recreational drug use, the presence of bilateral basal ganglia changes was a diagnostic clue that the patient was a heroin user.

\section{Conclusion}

The available history in patients presenting with loss of consciousness is often insufficient. Brain CT proved to be greatly beneficial for the evaluation of these patients. Opioid overdose should be suspected when bilateral basal ganglia lesions are present.

\section{Statement of Ethics}

Written informed consent was obtained from both cases for publication of this case report and accompanying images.

\section{Disclosure Statement}

The authors declare that they have no conflict of interest regarding the publication of this case report.

\section{Funding Sources}

The authors did not receive any external funding. 


\section{References}

1 Degenhardt L, Charlson F, Mathers B, Hall WD, Flaxman AD, Johns N, et al. The global epidemiology and burden of opioid dependence: results from the global burden of disease 2010 study. Addiction. 2014 Aug; 109(8):1320-33.

2 Schuchat A, Houry D, Guy GP Jr. New data on opioid use and prescribing in the United States. JAMA. 2017 Aug;318(5):425-6.

3 Seth P, Scholl L, Rudd RA, Bacon S. Overdose Deaths Involving Opioids, Cocaine, and Psychostimulants United States, 2015-2016. MMWR Morb Mortal Wkly Rep. 2018 Mar;67(12):349-58.

4 Bates DD, Gallagher K, Yu H, Uyeda J, Murakami AM, Setty BN, et al. Acute radiologic manifestations of America's opioid epidemic. Radiographics. 2018 Jan-Feb;38(1):109-23.

5 Lim CC. Magnetic resonance imaging findings in bilateral basal ganglia lesions. Ann Acad Med Singapore. 2009 Sep;38(9):795-8.

6 Yarid NA, Harruff RC. Globus pallidus necrosis unrelated to carbon monoxide poisoning: retrospective analysis of 27 cases of basal ganglia necrosis. J Forensic Sci. 2015 Nov;60(6):1484-7.

7 Andersen SN, Skullerud K. Hypoxic/ischaemic brain damage, especially pallidal lesions, in heroin addicts. Forensic Sci Int. 1999 May;102(1):51-9.

8 Iqbal I, Alam Khan MA, Ullah W. Can inhaled heroin be a cause of bilateral globus pallidus ischemic injury? Turk J Emerg Med. 2018 Nov;19(1):39-41.

9 Corré J, Pillot J, Hilbert G. Methadone-induced toxic brain damage. Case Rep Radiol. 2013;2013:602981.

10 Taheri MS, Noori M, Shakiba M, Jalali AH. Brain CT-scan findings in unconscious patients after poisoning. Int J Biomed Sci. 2011 Mar;7(1):1-5.

11 Jamshidi F, Sadighi B, Aghakhani K, Sanaei-Zadeh H, Emamhadi M, Zamani N. Brain computed tomographic scan findings in acute opium overdose patients. Am J Emerg Med. 2013 Jan;31(1):50-3.

12 Ramirez-Zamora A, Ramani H, Pastena G. Neurological picture. Bilateral pallidal and medial temporal lobe ischaemic lesions after opioid overdose. J Neurol Neurosurg Psychiatry. 2015 Dec;86(12):1383-4.

13 Geibprasert S, Gallucci M, Krings T. Addictive illegal drugs: structural neuroimaging. AJNR Am J Neuroradiol. 2010 May;31(5):803-8.

14 Corliss RF, Mandal R, Soriano BJ. Bilateral acute necrosis of the globi pallidi and rhabdomyolysis due to combined methadone and benzodiazepine toxicity. Am J Forensic Med Pathol. 2013 Mar;34(1):1-4.

15 Pearson J, Baden MB, Richter RW. Neuronal depletion in the globus pallidus of heroin addicts. Drug Alcohol Depend. 1976 Jun;1(5):349-56. 


\section{Case Reports in Acute Medicine}

\begin{tabular}{l|l}
\hline Case Rep Acute Med 2019;2:62-68 \\
\hline DOI: 10.1159/000503952 & $\begin{array}{l}\text { @ } 2019 \text { The Author(s). Published by S. Karger AG, Basel } \\
\text { www.karger.com/cra }\end{array}$ \\
\hline
\end{tabular}

Hassan et al.: Bilateral Basal Ganglia Lesions in Heroin Overdose

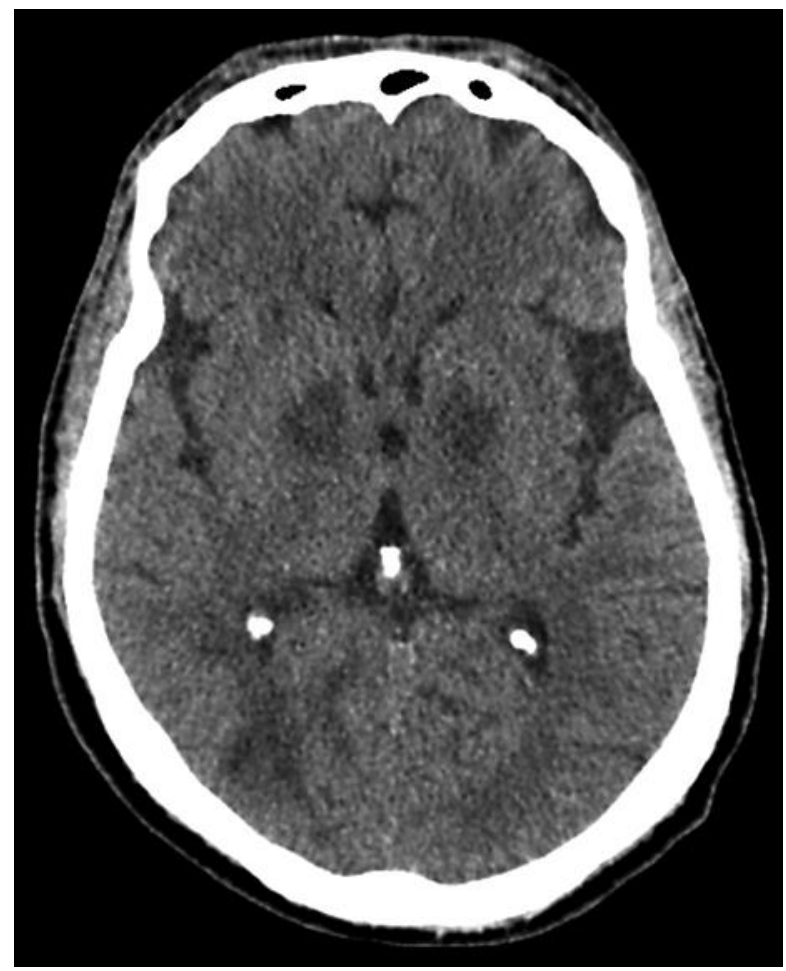

Fig. 1. Noncontrast axial computed tomography scan of the head of case 1 showing symmetrical bilateral hypodensities involving globi pallidi and genu of the internal capsule. 


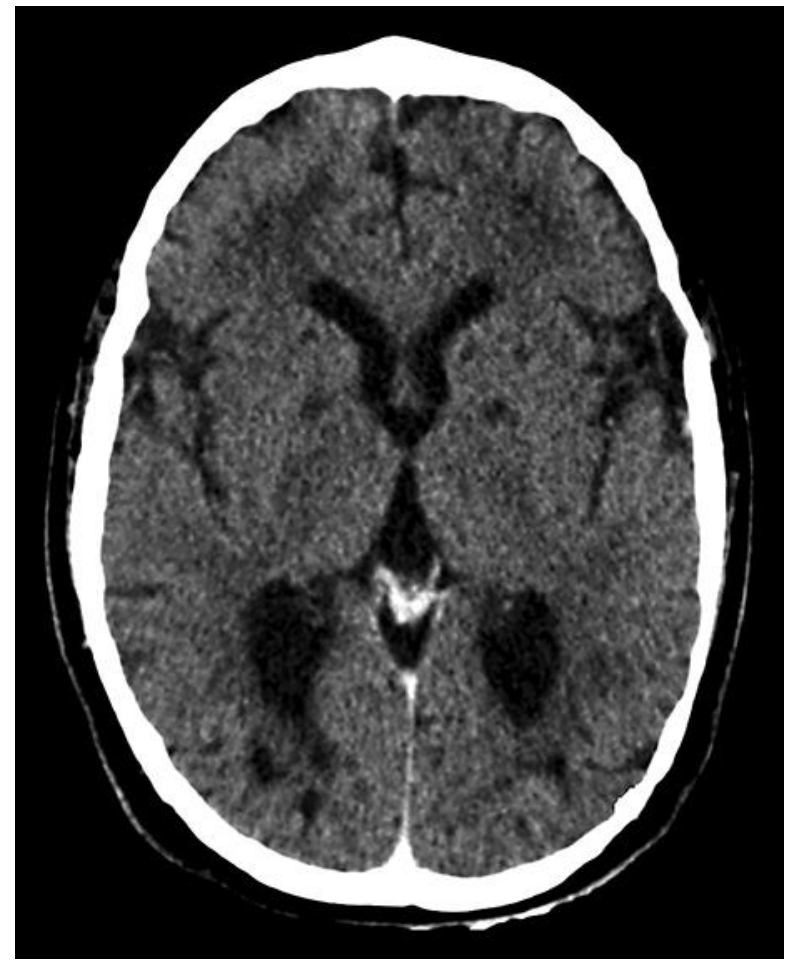

Fig. 2. Noncontrast axial computed tomography scan of the head of case 2 showing near symmetrical areas of subcortical and periventricular hypodensities scattered within the bilateral cerebral and cerebellar hemispheres, including the globi pallidi (cerebellum not shown). 